\title{
Role of physiological monitoring in educational organizations
}

\author{
Olga Shchkvirina*, Dinamutdin Misirov, Marina Akopyan, Irina Loseva \\ Southern Federal University, Rostov-on-Don, 344000, Russia
}

\begin{abstract}
An indicators analysis of the first-graders functional state and vegetative homeostasis obtained in the initial period of adaptation to the educational environment revealed gender differences in the rates of the adaptation process to new learning conditions. It has been established that girls' adaptation mechanisms turn on earlier than boys', with their similar orientation. The article provides an experimental justification of the indicators use legitimacy for heart rate variability to assess the functional state of the body in the acute period of adaptation to the educational environment.

The importance of teacher physiological literacy for the optimization of the educational process is substantiated. The results of the study are convincing evidence of the knowledge importance for the physiological foundations of the occurrence and course of physiological and mental processes, stable individual psycho-physiological characteristics for the organization of the learning environment, corresponding to the functional capabilities of the body.

This argument is also confirmed by many years of experience among master students key competencies development, which allow using such an objective criterion as the current functional state of the body to ensure, through the joint efforts of parents, teachers, psychologists, and doctors, the correction of the educational environment and psycho-physiological resistance of individual to it. The established differences in the dynamics of heart rate variability indicators for girls and boys can be used to form a health-saving program in school settings.
\end{abstract}

The results of the study allow us to conclude that the integration of systematic fundamental knowledge about the age dynamics of structure and function at various stages of education and training is facilitated by the introduction of the "Pedagogical Physiology" course for the educational process. During the implementation of educational projects, future teachers expand skills in developing innovative pedagogical projects for improving the educational institution functioning and models for their practical implementation, including forms and methods of work, assessment criteria, monitoring methods and development of individual, family, school, municipal and regional programs "Education and Health".

\footnotetext{
*Corresponding author: oishkvirina@sfedu.ru
} 


\section{Introduction}

One of the mechanisms for building a school-based health culture is the creation of a program to protect the health of children and adolescents. Acting as a promoter of the experience of healthy lifestyle implementation, teachers insufficiently use the knowledge of age and pedagogical physiology in organizing the educational environment. It is the knowledge of the child "in all respects" that gives rise to understanding that is the source of pedagogical science, the basis of teacher's creativity, the search for one's own ways in solving problems with individual students in the initial period of adaptation to learning (Krivolapchuk I.A., 2014).

According to physiological, hygienic and psychological research data, the intensification of the educational process without practical skills of teachers in organizing a personalityoriented educational process based on fundamental knowledge of age, gender and individual typological characteristics of a growing organism negatively affects the health status of students. The problem of diagnosing, preventing and effectively correcting the functional state of the schoolchildren's body in the process of adapting to educational activities remains both theoretically and practically significant (Krivolapchuk I.A. et al., 2016; Karantysh G.V. et al., 2017) and still are not enough studied (Sharapov A.N. et al., 2017; Kapilevich L.V. et al., 2011).

The feasibility of an individual approach in the upbringing and education of children does not require evidence. The initial period of systematic schooling is critical for the functional state and health for the child, and is reflected in the quality of learning and student behavior. Any adaptation to the activity is the output of the bio-system to a new level of functioning. Therefore, the full implementation of the urgent adaptation period is a prerequisite for the reliability of long-term adaptation.

\section{Research Methods and Methodologies}

To control the adaptation of students in school means to increase the resistance of their body, which forms the ability to adequately respond to various influences (Sitdikov F.G. et al., 2006). Currently, one of the non-invasive methods to assess the adaptive reactions of the body is the analysis of the wave structure of the heart rhythm (Krivolapchuk I.A., 2018 et al.; Gorelik V.V., 2019). In this regard, the goal of this study is the physiological assessment of changes in the first-graders functional state of the body during the urgent adaptation period during the 3rd, 5th, 8th weeks of training. Monitoring diagnostics was carried out for 33 firstgrade schoolchildren (16 boys and 17 girls) at primary school No. 24, Rostov-on-Don with the help of the specialized hardware-software complex "Varicard 2.51" in the mornings.

\section{Research results}

It has been shown that the features of the autonomic nervous system (tone, reactivity, balance) are one of the leading parameters that determine the effectiveness of activity (Krivolapchuk I.A. et al., 2018). The analysis of the research results studying the dynamics of the functional state of first-graders indicates significant gender differences in the development of the adaptation process to the educational environment. When the body experiences oxygen starvation, heart rate, cardiac output, and myocardial contractility tend to increase. At all stages of the examination, heart rate indices for boys and girls alone do not have significant differences, which is due to the high individual variability of the data. The type of body reaction to orthostasis is a reflection of the adaptive capacity of the body. 
Significant increases in heart rate during an orthostatic test were found for boys during the third $(\mathrm{t}=3.43 \mathrm{p}<0.01)$ and the eighth $(\mathrm{t}=2.05 \mathrm{p}<0.05)$ weeks of the examination, and for girls during all periods of the examination $(\mathrm{t}=2.94 \mathrm{p}<0.01 ; \mathrm{t}=3.11 \mathrm{p}<0.01 ; \mathrm{t}=2.56 \mathrm{p}$ $<0.05$ ). The high reactivity of regulatory systems of girls, especially during the fifth week of training, reflects the stressful period of urgent adaptation, as well as the adaptive plasticity of their body. Significant reductions in the majority of girls examined heart rate of orthostasis $(\mathrm{t}=2.87, \mathrm{p}<0.01)$ from the 5 th to the 8 th week of training indicates a productive interaction of the body with the environment, i.e. heart rate changes are reversible, and learning activity that induces the body's adaptive response corresponds to the functional capabilities of their body.

The dynamics of regulatory systems activity indicators (RSAI) for boys in the dynamics of observation only $25 \%$ of boys during the third week and $28.6 \%$ during the eighth week of training revealed sufficient functional (adaptive) capabilities of the body, the adequacy of responses, the state of the optimal operating voltage of regulatory systems.

Large group of examined boys during all periods of the examination $(50 \%, 50 \%, 57.2 \%)$ has a pre-nosological functional state, which indicates activation of the adaptation process associated with the voltage of regulatory systems for this group of children. Under prenosological conditions, the body needs additional functional reserves to adapt to the conditions of the educational environment; it requires a moderate tension of regulatory mechanisms, which reduces the body's adaptability to stresses. The pre-nosological functional state occurs under various stressful effects, but in the presence of sufficient functional reserves of the body, adaptation proceeds successfully. With increased expenditure of reserves during the course of adaptation mechanisms tension, pre-nosological conditions can go into pre-morbid (pre-illness) and then into pathological (disease) conditions.

During research dynamics of the boys' body functional state a decrease in functional reserves (pre-morbid state) was revealed for $12.5 \%$ during the third week, $37.5 \%$ during the fifth, $14.3 \%$ during the eighth week of training. The presence of such a body functional state at rest is a sign of an inadequate response to the influence of environmental factors, and constant stress on the pre-morbid state leads to an accelerated expenditure of vital resources and the development of diseases. Disruption of adaptation mechanisms was revealed during the third and fifth weeks in a small group for boys of the first class $(14.3 \% ; 12.5 \%)$. The absence of boys with maladaptation during the eighth week of the examination indicates the adequacy of the educational environment organization to the functional capabilities of the body for this group of boys.

The results of the survey of the regulatory systems activity indicator for girls showed good adaptability to learning conditions with optimal tension of regulatory mechanisms found for $30.7 \%$ during the third week, $7.7 \%$ during the fifth week and $50 \%$ - during the eighth week of the initial period of adaptation to systematic schooling.

The pre-nosological state bordering between norm and pathology in the norm-load of regulatory mechanisms was detected for $38.4 \%$ during the third week, $69.2 \%$ during the fifth week and $33.3 \%$ during the eighth week of the adaptation process. It is important to note that the tension of regulatory mechanisms is an unstable period of adaptation. A pre-morbid state, indicating maladaptive states, with severe fatigue, autonomic dysfunctions, when stresses go beyond the adaptive capacity of the body, was detected for $30.7 \%$ of girls during the third week, $23 \%$ during the fifth week and $16 \%$ during the eighth week. Girls with a failure of adaptation, i.e. having a depletion of vitality, are not revealed in all periods of the study during the adaptation process.

Children functional state and adaptive capabilities of primary school age largely determines the state of autonomic homeostasis. It has been established that a constant stress state caused by the conditions of preparation and the beginning of schooling for children 6-7 
years old is the trigger mechanism of cardiovascular diseases. According to numerous studies, the regulation of heart rhythm for children is determined by age, gender, and individual typological characteristics (Krivolapchuk I.A. et al., 2016; Longin E. et al., 2009; Montano N. et al., 1994). A negative effect on the body functional state and stress resistance for primary schoolchildren of a sympathetic system high tone and hypothalamic structures sympathetic tuning has been established (Everly G. et al., 2013; Quas J.A. et al., 2014 and others). It is known that in an equilibrium state of the sympathetic and parasympathetic departments of the autonomic nervous system (ANS), the body has good resistance to the effects of stimuli of various strengths.

\section{Research Results Discussion}

The research results revealed the functional synergy of the ANS departments in the regulatory mechanisms of heart rhythm to a greater extent for girls. The data show that at rest, the number of girls with a vegetative balance of regulation almost doubles from the 3rd to the 8 th week of the survey $(31 \%, 58 \%)$. That is convincing evidence of a good adaptation to the educational process for this group of first-graders.

The moderate prevalence of the vagus nerves influence at rest for $39 \%$ of girls during the third week of adaptation gives great reserve possibilities for the heart of their body to intensify their work, which is ensured either by an increase in the influence of the vagus, or by a decrease in the activity of the sympathetic nerves. A.A. Ukhtomsky noted (Utomsky A.A., 1933) that the essence of the relationship between the sympathetic and parasympathetic nerves lies in the compensatory alignment of the vagal effect with the sympathetic effect. Moderate activity of the parasympathetic regulatory link indicates a good efficiency in the restoration of functional reserves spent on adaptation changes. For this group of girls, the parasympathetic division of the ANS makes the largest contribution for the heart rhythm regulation, which reflects a good physical condition and stress resistance of the body. Sympathetic activation indicates the inclusion of adaptation mechanisms. For $31 \%$ of girls, these mechanisms are actively working during the 5th week of training. Hypersympathetic activation for $15 \%$ of girls during the 3 rd week and $8 \%$ during the 5 th week of the adaptation process indicates a decrease in the reserve capacity of the autonomic regulation of heart rhythm for this group of girls.

The autonomic homeostasis dynamics during adaptation for boys suggests that a vegetative balance was found in a small group of boys at all periods of the survey $(12.5 \%$; $12.5 \% ; 14.3 \%)$.

During the third week of training, the majority of boys at rest revealed a predominance of parasympathetic tone ( $25 \%$ - pronounced predominance and $37.5 \%$ - moderate), and for $25 \%$ of boys - moderate predominance of the sympathetic ANS. Boys with a pronounced predominance of the sympathetic part of the autonomic nervous system in the survey were not detected. During the fifth week of adaptation, $25 \%$ of the examined boys retain the pronounced predominance of the parasympathetic nervous system, the group of children with a moderate prevalence of the parasympathetic ANS increases to $50 \%$, i.e. more than half of the boys in the survey group (75\%) have a sufficient functional reserve to adapt to new learning conditions. For $12.5 \%$ of boys, a moderate predominance of the sympathetic nervous system remains. At the eighth week of education for the first grade, the main group of boys $(57 \%)$ has a moderate prevalence of the sympathetic division of the ANS, which indicates the active inclusion of adaptation mechanisms to the educational environment.

Thus, the study reveals the inclusion of adaptation mechanisms due to the sympathetic tone of the ANS, as an adaptation reserve for most children of both sexes, however, for most girls of the examination group they included during the 5th week of training, and for boys later during the 8th week of training. A high level of vegetative changes for girls may indicate 
greater functional capabilities of their body compared to peers. Changing the vegetative balance is necessary for expanding and forming the adaptive range of a growing organism. The functional state of the schoolchildren body determines not only its adaptive capabilities, level of health, but also the success of school education. Researches show that for healthy children with good resistance, regardless of the effects of stimuli, the sympathetic and parasympathetic divisions of the ANS are in equilibrium. But the growth and development rates are individual. Therefore, at each stage of ontogenesis, regulatory mechanisms have their own characteristic features associated with both the processes of adaptation to new environmental conditions and the processes of growth and development. In this regard, for educational environment organizing at the initial period of adaptation to the modern educational process, not only age and individual, but also gender characteristics of schoolchildren should be taken into account.

Higher functional capabilities of girls' regulatory mechanisms, providing earlier adaptive rearrangements of their body to the educational environment at the initial period of schooling, indicate more mature cholinergic effects on the myocardium at the studied age range for girls, i.e. they are ahead of boys by their biological age (Krivolapchuk I.A., 2014).

The research results allow us to conclude that during the initial period of adaptation to the educational process, first-graders are at high risk of developing autonomic dysfunction with inadequate functional capabilities of the schoolchildren body during educational environment organizing.

The integration of systematic fundamental knowledge about the age dynamics of structure and function, reserve capabilities, adaptation mechanisms and the psycho-physiological functions of a growing organism at various stages of ontogenesis with the practice of upbringing and training is facilitated by the introduction of the "Pedagogical Physiology" course in the educational process. During the implementation of educational projects, future teachers develop skills during innovative pedagogical projects creation for improving the educational institution's health and model of their practical implementation, including forms and methods of work, assessment criteria, monitoring methods and the development of individual, family, school, municipal and regional programs "Education and health."

Conducted research helps to understand the peculiarity of each age period of ontogenesis, to trace the dynamics of its basic laws, to understand the physiological mechanisms of learning difficulties. The acquisition of systematic skills in using the knowledge of children's physiology in education and managerial practice makes this knowledge a vital necessity. So, according to the research, a steady vegetative tone (vegetative balance) is the key to successful adaptation and successful study. On the contrary, a very high sympathetic tone and high lability of the vegetative tone reduce the quality of academic performance, and, consequently, the mental stability of first-graders.

\section{Conclusion}

In conclusion, it should be noted that in the process of research, the master student of education acquires the skills of physiological and hygienic analysis of the educational environment. It is important for future teachers to form a motivated attitude to the psychophysiological testing of the functional state for the human body as a universal method of objective assessment of the quality for the "organism-environment" system in general and "organism-educational environment" in particular. It is important to understand that the reactions of the child's body to training loads often exceed the physiological norm and are associated with the development of new adaptive mechanisms in the functioning of visceral systems, and this must be taken into account when optimizing the educational process. Physiological monitoring allows us to assess resistance to "school" stress, to establish physiological mechanisms that do not allow schoolchildren to study well. 
Physiological monitoring data serve as an indicator of the functional state of the body and the quality of its living environment, on the basis of which an adequate system of primary prevention and correction of school-significant functions and systems of vegetative support is selected, as well as the design and correction of the educational environment and educational process. At the same time, the mechanism of physiological testing, its assessment and practical application should be in the hands of a teacher who knows children's physiology well and is constantly growing in this direction. In this regard, the functional state and health of the adult organism (parents and teachers) is considered as one of the leading risk factors for the same condition for children and adolescents, and their physiological literacy is a condition for the realization of the child's right to individual development.

\section{References}

1. V.V. Gorelik, S.N. Filippova New physical education at school: corrective and healthimproving use of physiological indicators for students as markers of physical development and health disorders, Man. Sport. The medicine, 19 (1), $42-49$ (2019). DOI: 10.14529 / hsm190106

2. L.V. Kapilevich, V.G. Shilko, A.V. Kabachkova, Physiological monitoring and monitoring of health-saving activities in the process of physical education of students, Bulletin of Siberian medicine, 4, 76-81 (2011).

3. G.V. Karantysh, D.N. Misirov, A.M. Menzheritsky, L.M. Dmitrenko, T.V. Lastavchenko, The dynamics of the functional indicators of the body in adolescents with different levels of physical performance. Problems of modern teacher education, Ser. Pedagogy and psychology, Scientific journal, Yalta: RIO GPA, 53 (10), 230-238 (2016).

4. K.I. A.rivolapchuk, M.B. Chernova, Functional state of children of 6-8 years of age during testing under the influence of various types of stress-strain loads, Neuroscience and physiology of behavior, 48 (6), 747-757 (2018).

5. I. A. Krivolapchuk, Features of the physical condition of boys and girls of preschool age, MedicinadelloSport, 67 (2), 241-250 (2014).

6. I.A. Krivolapchuk, M.B. Chernova, The functional state of schoolchildren aged 6-7 with cognitive load, Almanac "New Studies", 2, 36-44 (2016).

7. N.V. Litvinenko, Adaptation of students in critical periods of development to the educational environment, Abstract. dis. ... doctor. psychol. sciences. Samara, 2009.

8. F.G. Sitdikov, M.V. Shaikhelismanova, A.A. Sitdikova, The functional state of the sympatho-adrenal system and the features of the autonomic regulation of heart rate for younger students, Human physiology, 6, 22-27 (2006).

9. A.N. Sharapov, S.B. Dogadkina, L.V. Rubleva, G.V. Kmit, V.N. Bezobrazova, Functional state of the cardiovascular system of 13-year-olds with different types of autonomic nervous regulation, Human Physiology, 43 (2), 31-42 (2017).

10. A.A. Ukhtomsky, 15 years of Soviet physiology: monograph (OGIZ-Medgiz, Leningrad, Moscow, 1933).

11. G. Everly, J. Latin, A Clinical Guide to the Treatment of the Human Stress Pesponse (Springer, NY, 2013).

12. E. Longin, C. Dimitriadis, S. Shazi, T. Gerstner, T Lenz, S. König, Autonomic nervous system function in infants and adolescents: impact of autonomic tests on heart rate variability, PediatrCardiol, 30 (3), 311-324 (2009).

13. N. Montano, T.G. Ruscone, A. Porta et al., Power spectrum analysis of hear trate variability to assess the changes in sympathovagal balance during graded orthostatic, 
Circulation, 90 (4), 1826-1831 (1994).

14. J.A. Quas, I.S. Yim, T.F. Oberlander, D. Nordstokke, M.J. Essex, J.M. Armstrong, N. Bush, J. Obradović, W.T. Boyce, The symphonic structure of childhood stress reactivity: patterns of sympathetic, parasympathetic, andadrenocortical responses to psychological challenge, Dev Psychopathol, 26 (4), 963-982 (2014).

15. D. Shulhan, H. Scher, J. Furedy, Phasic Reactivity to Psycholocigical Stress as a Function of Aerobic Fitnes Level, Psychophysiology, 23 (5), 562-566 (1986). 